It became clear in the discussion that many of the governments of the Asian countries are fully alive to the dangers of population-growth and are encouraging research on these topics. Such research has the aim of providing better data to help in the understanding of these problems and also in devising measures which would help to slow down the rate of increase. A number of important and interesting communications described field-studies in population in progress in India, Indonesia, Burma and Japan and in some parts of Africa. The application of sampling techniques to demographic research in particular has proved very fruitful. In India, the government is encouraging research on methods of birth control, and also conducting a field-survey, with the technical assistance of the United Nations, on the efficacy of the 'rhythm' or 'safe-period' method of birth control as a check on population in some Indian villages. It was clear that in societies in which there is a high degree of illiteracy, the introduction of even the simplest methods of birth control would be fraught with difficulty, although there was considerable evidence of a latent demand for it on the part of parents who have already had three or more children.

In contrast there were Prof. Y. Koya's findings in Japan. 'Ihere, the dissemination of contraceptive information in a number of fishing villages has had the effect of reducing the general fertility-rate in those areas by about one-third within a fairly short space of time. The population of Japan is, of course, more literate than that of India; but the success of the experiment astonished the Japanese themselves, who were surprised by the absence of a demand for large families which they thought implicit in the Japanese system of values. Interesting facts were also presented regarding the response to the recent Japanese law which has legalized abortion on sociomedical grounds. Recent Japanese experience was cited in support of the views of those participants who believe that increasing industrialization would ipso facto bring with it lowered fertility. It was made clear, however, that even if fertility in the Eastern countries were to decrease as a consequence of industrialization and economic progress, the present age-structures of those populations are such that in any event considerable increases may be expected in the near future.

A forecast of the U.N. Population Division for the population of the world as a whole estimated that by 1980 the figure would lie between 3,300 and 4,000 million people, against an estimate of 2,500 million in 1950. This estimate was put forward with all due caution. There are many areas of the world for which the data are still rudimentary, and if recent reports putting the population of China at 630 million prove correct, even the basic estimate for 1950 may be in error by as much as $7 \frac{1}{2}$ per cent. In spite of this, it was considered valuable to have an extrapolation of present trends in different areas prepared by an impartial and expert body like the United Nations.

A word or two may be said about the attitude of participants from Communist countries. The absence of Chinese demographers has already been com. mented upon and was keenly felt in view of recent reports in the Press on the results of the latest Chinese enumeration. It was clear that there are considerable differences between the points of view of the Communist demographers and their Western and Asiatic colleagues. The Communists regard continued population-growth as a sign of a healthy society and refuse to be perturbed by the prospect of continued pressure of population upon resources. In a communication to the Conference, Mr. Ryabushkin, of the U.S.S.R., wrote: "The Neo-Malthusians proposed to bring the number of population into conformity with the level of production. But an elementary logic suggests that on the contrary levels of production should be brought into conformity with the number of population, because it is production that exists for population, but not population for production. And that production can be repeatedly increased in a relatively short period of time is evident from the historical experience of the Soviet Union."

This point of view, which implicitly assumes that natural resources are unlimited, has in practice aligned the Communists with the Roman Catholic Church in their attitude towards birth contirol. Neo-Malthusianism is a term of abuse, contraception is discouraged and it is not clear to what extent contraceptives are made available to those who desire them on other than medical grounds. Maternity and child-welfare services are available to mothers and high fertility is encouraged. But it is by no means clear from the addresses delivered at the Conference to what extent the right of individuals to decide on the size of family that they would like to have is recognized in Communist countries.

In concluding, tribute should be paid to the success of the administrative arrangements for the Conference, which was held in the building of the Food and Agriculture Organization, and to the skill of the interpreters, who were successful in providing simultaneous translation of addresses which were frequently highly technical into English, French, Russian and Spanish.

E. GREBENIK

\section{POST OFFICE ENGINEERING RESEARCH STATION, DOLLIS HILL OPEN DAY}

$T$

H.E Post Office Engineering Research Station, Dollis Hill, was open to visitors from the universities, industry and government departments on September 24, the Press having been invited the previous day. The functions of the Station, its staffing and its relationships with the telecommunications industry remain much the same as on the last open day (see Nature, 168, 1022; 1951), though the buildings have since been increased, notably by one which provides a ground floor of nearly twenty thousand square feet devoted mainly to machine shops, an upper floor for drawing offices and part of another floor to a suite of laboratories devoted to thermionics. The individual problems being undertaken, whether basic studies or the development of new techniques or equipments to meet specific requirements, have, however, changed considerably, as the following descriptions of a few of the eighty exhibits presented will show.

The application of electronic techniques to telephone switching was shown in a private automatic branch exchange, devoid of electromechanical parts ; it has an ultimate capacity of ninety-nine lines, exchange and extension, of which forty are now being connected. The switches use time-division-multiplex transmission, each line being allocated a fixed 
position, of duration 1 usec., in a continuously repeated cycle of $100 \mu$ sec., for the transmission and reception of speech currents. Registers and markers control the switches. The former receives the numerical information from the calling subscriber and stores it on gas discharge tubes before passing it to the marker which causes the called line to be tested and, if free, to be connected to the electronic equivalent of a cord circuit to which the calling line was joined when the call originated. A register is in service for as long as it takes a subscriber to dial, the marker for about 20 m.sec.

The Station has designed an electronic digital computer called MOSAIC and installed it at an establishment of the Ministry of Supply. The computer could be shown, therefore, only by way of photographs of key parts. It uses a pulse repetition frequency of about $600 \mathrm{kc} . / \mathrm{s}$.; storage is in ultrasonic delay lines with a full capacity of 1,030 words (instructions) and numbers, each of 40 bits, that is, each number corresponds to twelve digits in the scale of ten. A four-address system is used : each instruction to the machine defines the location of the two numbers to take part in the next arithmetical step, the destination of the answer and the location of the next instruction. The instructions and the numbers to be operated on are fed in, and the answers fed out, on punched cards.

Recently new attempts have been made to extract the essential information in speech in such a way as to enable it to be conveyed over a channel of bandwidth much less than that conventionally used in telephony $(\sim 3,000 \mathrm{c.} / \mathrm{s}$.$) . It is well known that$ speech can be synthesized sufficiently well by using only six slowly varying parameters - the level and pitch of the larynx tone, the level of hiss excitation and the frequencies of the three major resonances (formants) in the vocal tract-but until now it had not been possible to extract the last three instantaneously from speech. Progress was demonstrated, however, with equipment which sets up a spectral display differentiated with respect to frequency to find the resonances. The display is followed by circuits which generate the control signals of a synthesizer. The six signals may require a total bandwidth of only $180 \mathrm{c} . / \mathrm{s}$, to which must be added, however, that required by the filter designer to ensure satisfactory assembly and separation.

Transistors have yet to make any impact on the telegraph and telephone systems in Great Britain, but some simple line amplifiers and a quartzcontrolled oscillator using germanium junction transistors showed some of the advantages of small size and power consumption which may later be obtainable. The great and almost immediate susceptibility of fully exposed germanium junction diodes and triodes to water vapour at room temperature and at a pressure of about $1 \mathrm{~cm}$. of mercury was demonstrated; the maximum voltage which the diode or the collector-base junction could sustain fell drastically-recovering equally rapidly on sudden lowering of the vapour pressure to below $1 \mathrm{~mm}$. For reliability, therefore, water vapour must not be allowed access to the active element.

Two new pieces of spectrographic equipment were on show. A compact source unit can produce a d.e. arc, a high-voltage spark and some triggered ares, and a projector-comparator enables spectra on one or two plates to be seen and compared at a magnification of twenty times for the purposes of identification and semi-quantitative analysis.
Magnetic materials, so widely used in telecommunications, have been the subject of several developments. Nickel-iron strip as thin as 0.00015 in. has been rolled by a novel technique; its low-field permeability can be as high as 20,000 and its coercive force as low as 0.025 oersted after suitable treatment; thicker material (for example, 0.002 in.), similarly treated, can have values of 75,000 and 0.0075 oersted.

Repeaters (amplifiers) for inclusion in submarine cables have engaged more and more attention since 1945 (the first one was laid by the British Post Office in the Irish Sea in 1943) and have continually thrown up new problems as submarine schemes became more ambitious. The practical solutions to some, such as that of making the gland between cable and repeater capable of preventing the entry of water at the pressures encountered at depths of a mile or two and that of making reliable all the components (the failure of any one of which could, without other safeguards, involve a cable ship in many days of work) often entail meticulous attention to detail with no spectacular results; they are, however, being obtained. Ingenuity in the electrical design of the amplifier has eased some of the problems. Much improved accuracy in the measurement of the electrical properties of submarine cables has been necessary to engineer some of the longer systems; when obtained, it has brought to light new effects affecting the properties. Progress towards an oxidecathode valve of high performance and long life (perhaps twenty years) has been made using a passive platinum core, the fundamental behaviour of which is becoming understood, rather than the active nickel core so widely used in commercial valves. Careful attention must be continuously given to pre-processing, assembly and vacuum techniques.

British deep-sea repeaters, though still housed in rigid cases, are streamlined and more easily handled during laying than the earlier shallow-water types. Telemetering devices fitted within a dummy repeater, for measuring shocks, spin, inclination and pressure, have been developed in order to investigate the conditions encountered between the time a repeater enters the water and when it finally settles on the sea bed.

The Post Office continues to give much attention to the methods of producing quartz resonators ; the Essen rings which the Post Office has recently supplied as standards to the Greenwich, Ottawa and Naval (Washington, D.C.) Observatories and to the National Physical Laboratory (Teddington), the National Research Council of Canada (Ottawa) and the National Bureau of Standards (Washington, D.C.) illustrate some of the newer techniques. The quartz is now cut by an annular saw which reduces waste and surface damage and permits thinner wafers to be specified.

Quartz continues to play a part in narrow-band filters. At the same time, coil-condenser filters, so widespread in multi-channel telephony, still find new uses in radio engineering. The output stages of $a$ radio transmitter generate harmonics which, if the fundamental frequency is up to, say, $20 \mathrm{Mc}$./s., may interfere with the local reception of television, particularly in fringe areas ; a low-pass coil-condenser filter capable of dissipating that fraction of the transmitter power at the fundamental frequency which it absorbs (perhaps $0 \cdot 3$ per cent or up to 50 W.), inserted between the output stage and the 
aerial, can sufficiently attenuate the harmonies. Filters play an increasing part in multi-channel microwave systems; Darlington's and Cocci's methods of network synthesis are being successfully applied to designs involving irises and posts in rectangular wave-guides.

The transmission of telephony and television at microwave frequencies could not be successfully undertaken without precision measuring equipment. Group-delay distortion of a frequency-modulated multi-channel telephony system results in intermodulation products of which little can be tolerated. The distortion introduced by filters, travelling-wave tube amplifiers and other components, over a bandwidth of $50 \mathrm{Mc} / \mathrm{s}$. centred at about $4,000 \mathrm{Mc} . / \mathrm{s}$., can now be displayed on a cathode-ray tube with a sensitivity of $1 \mathrm{~cm} . / \mathrm{musec}$.

Equipment for long-distance radio communication in the 4-30 Me./s. band is judged in part by its ability to combat the fading and multipath effects characteristic of ionospheric propagation. Because testing in service is slow and inconvenient, a fading machine simulating the natural effects is a great asset. An early machine, producing frequencyselective fading by combining two or three differently delayed signals of constant amplitude, but varying relative phase, has recently been modified so that the differently delayed signals individually fade in a near-random way, more closely simulating field conditions.

\section{NEWS and VIEWS}

\section{Nobel Prize in Physics :}

Prof. Max Born, F.R.S.

Prof. Max Born, who has been awarded a share of the Nobel Prize in Physics for 1954, is known for many contributions to modern theoretical physies, particularly to the development of quantum mechanics and to the theory of crystals. The work mentioned specifically in the announcement of the Nobel award is the interpretation of the wave functions as probabilities for the positions of particles, a vital step in the development of the modern view of the relations between particle and wave aspects of atomic theory. This dates from a period when, in collaboration with Heisenberg and others, Born took an important part in the development of quantum mechanics, which form one of the two independent approaches to modern quantum theory. Other important work of that period, which has become classical, includes his use of perturbation theory for scattering problems and the Born-Oppenheimer theory of molecules. The theory of crystal lattices has formed another continuing interest, from the earliest papers which showed the basic ideas of lattice vibrations and their relation to the interatomic forces, to several treatises covering all aspects of the theory. Born has also taken an active interest in many other aspects of quantum theory. His effect on theoretical physics should, however, be measured not only by his own papers, numerous as they are, but also by the work of his pupils. His school at Göttingen was for a long time one of the most important centres to which young theoreticians went to find guidance and inspiration and to be infected with that enthusiasm and optimism which characterize Born's own work. The men who worked with him later at Cambridge and Edinburgh also include many who have made their mark on modern physics.

Prof. W. Bothe

Prof. W. Вотне, of the University of Heidelberg, who shares the 1954 Nobel Prize in Physics with Prof. Born, is known for many important contributions to modern physics. The best known of these include his introduction of coincidence methods into counting techniques and the work in which, together with Geiger, he applied the coincidence method to the Compton effect and showed that the conservation laws are satisfied in each individual event and not merely on the average. This was fundamental for the interpretation of atomic processes. With Kohlhörster he applied coincidence methods to the study of cosmic rays and thus helped to establish the presence of penetrating charged particles. Later, in the course of systematic work on nuclear reactions, he discovered the unusual radiation, emitted by beryllium under $\alpha$-particle bombardment, which was later identified as neutrons. Other fields in which he did pioneer work include the study of hard $\gamma$-rays and the nuclear photo-effect produced by such rays and the problems of multiple scattering of electrons and of neutrons. On problems of scattering he has not only done experimental work of great importance but has also made valuable contributions to the theory.

\section{Nobel Prize for Chemistry: \\ Prof. Linus Pauling, For.Mem.R.S.}

The Nobel Prize for Chemistry for 1954 has been awarded to Prof. Linus Pauling, of the California Institute of Technology. This award will be acclaimed by chemists of all kinds, for Prof. Pauling's contributions have brought about a revolution in the entire field of chemistry. His early work was concerned with the determination of crystal structures by X-ray crystallography, an interest which he has retained; his recent views on the constitution of proteins are based largely on detailed crystallographic analyses of amino-acids and peptides. The study of crystal structure led him to consider the nature of chemical bonds, and already in 1926 he published a remarkable paper on the structures of benzene and its analogues in terms of the Bohr atom. This, however, was soon followed by an impressive series of papers in which the new methods of wave mechanics were applied to chemical problems of all kinds, and in which the resonance theory was developed as a general qualitative approach to such problems. This work led to the recognition of additivity of bond energies and covalent radii in unconjugated molecules (that is, those with a unique classical structure), to the concept of resonance energy and intermediate bond character in conjugated molecules, and to the concept of electronegativity as a factor in determining covalent bond energies. Prof. Pauling's original tables of bond energies, covalent radii and electronegativities are still widely used, and later work has altered them only in detail. Many of these covalent radii were deduced from bond-lengths measured in his laboratory by the newly developed and difficult technique of electron diffraction. In recent years Prof. Pauling has made further notable contributions in fresh fields : a new theory of the metallic state, a theory 